\title{
CAUCHY-BINET TYPE FORMULAS FOR FREDHOLM OPERATORS
}

\author{
Grażyna Ciecierska \\ Faculty of Mathematics and Computer Science, University of Warmia and Mazury in Olsztyn \\ Olsztyn, Poland \\ grac@matman.uwm.edu.pl
}

Received: 19 September 2016; accepted: 4 June 2017

\begin{abstract}
Suppose $A \in L(Y, Z), B \in L(X, Y)$ are Fredholm operators acting in linear spaces. By referring to the correspondence between Fredholm operators and their determinant systems, we derive the formulas for a determinant system for $A B$ which are expressed via determinant systems for $A$ and $B$. In our approach, applying results of the theory of determinant systems plays the crucial role and yields Cauchy-Binet type formulas. The formulas are utilized in many branches of applied science and engineering.
\end{abstract}

MSC 2010: 47A53, $15 A 15$

Keywords: Fredholm operator, determinant system, reflexive generalized inverse, Cauchy-Binet theorem

\section{Introduction}

The purpose of this paper is to exhibit a method of construction of a determinant system for a product of arbitrary linear Fredholm operators acting between linear spaces. The method is based on tools of the determinant theory created by Leżański [1], developed and modified by Sikorski [2-4] and Buraczewski [5, 6].

We address the problem of how to express a determinant system for product $A B$ of Fredholm operators $A: Y \rightarrow Z$ and $B: X \rightarrow Y, X, Y, Z$ being linear spaces over the same field (real or complex), in terms of determinant systems for $A$ and $B$. In the derivation of the main result we use some ideas presented in [7] for Fredholm endomorphisms and extend them to Fredholm operators acting between arbitrary linear spaces. Since the method proposed in the paper is purely algebraic, we dispense with assumptions related to a topological structure of linear spaces involved. The formulas, obtained as a direct and constructive solution to the above mentioned problem, are generalizations of the classical Cauchy-Binet formula [8-10], which states that if $A$ and $B$ are two matrices over field $F$ of sizes $n \times m$ and 
$m \times n$, respectively, with $n \leq m$, then $\operatorname{det}(A B)=\sum_{p} \operatorname{det}\left(A_{p}\right) \operatorname{det}\left(B^{p}\right)$, where the sum is taken over all increasing sequences $p=\left(p_{1}, p_{2}, \ldots p_{n}\right)$, with $p_{i} \in\{1, \ldots, m\}$, and $A_{p}\left(B^{p}\right)$ is $n \times n$ submatrix of $A(B)$ obtained by deleting all columns (rows) except these with indices in $p$. When $n=m$, the formula becomes the well-known product formula $\operatorname{det}(A B)=\operatorname{det}(A) \operatorname{det}(B)$ for determinants. The Cauchy-Binet formula plays an important role in studies of determinants, permanents and other classes of matrix functions. An increasing interest in its applications in many branches of applied science, such as matrix analysis and engineering [11-13], is a motivation of the paper. It is worth emphasizing that, so far, many considerable contributions to generalizing the Cauchy-Binet theorem have been made [14-17]. In our approach, the proposed generalization to Fredholm operators is based on the correspondence between any Fredholm operator and its determinant system. We also make use of analogues of the Laplace expansion formula that are available for terms of determinant systems.

\section{Preliminaries}

In this section we recall the main notions and facts concerning the determinant systems theory and we fix the notation [3-6, 18-20].

Suppose $(\Xi, X),(\Omega, Y)$ and $(\Lambda, Z)$ are pairs of conjugate linear spaces (over the real or complex field $F$ ) with respect to scalar products $I$ on $\Xi \times X, J$ on $\Omega \times Y$ and $K$ on $\Lambda \times Z$, respectively, satisfying the cancellation laws [6]. Elements $\xi \in \Xi$ and $x \in X$ are called orthogonal if $I(\xi, x)=\xi x=0 ;$ moreover, $X_{0}{ }^{\perp}=\left\{\xi \in \Xi: \xi x=0\right.$ for all $\left.x \in X_{0}\right\}$ and $\Xi_{0}{ }^{\perp}=\left\{x \in X: \xi x=0\right.$ for all $\left.\xi \in \Xi_{0}\right\}$ for given subsets $X_{0} \subset X$ and $\Xi_{0} \subset \Xi$. Denote by $D\left(\begin{array}{ll}\xi_{1}, \ldots, & \xi_{\mu} \\ y_{1}, \ldots, & y_{m}\end{array}\right)$ the value of a $(\mu+m)$-linear functional $D: \Xi^{\mu} \times Y^{m} \rightarrow F$ at a point $\left(\xi_{1}, \ldots, \quad \xi_{\mu}, y_{1}, \ldots, y_{m}\right)$. $D$ is said to be bi-skew symmetric if it is skew symmetric both in variables $\xi_{1}, \ldots, \xi_{\mu}$ and $y_{1}, \ldots, y_{m} ; b s s_{\mu, m}(\Xi, Y)$ stands for the set of all bi-skew symmetric functionals on $\Xi^{\mu} \times Y^{m}$. We call $D$ an $(\Omega, X)$ - functional on $\Xi^{\mu} \times Y^{m}$ if for arbitrary fixed elements $\xi_{1}, \ldots, \xi_{i-1}, \xi_{i+1}, \ldots, \xi_{\mu} \in \Xi$ and $y_{1}, \ldots, y_{m} \in Y$ there exists an element $x_{i} \in X$ such that $\xi x_{i}=D\left(\begin{array}{ccc}\xi_{1}, \ldots, & \xi_{i-1}, \xi, \xi_{i+1}, \ldots, & \xi_{\mu} \\ y_{1}, & \ldots, & y_{m}\end{array}\right)$ for every $\xi \in \Xi$ $(i=1, \ldots, \mu)$ and for arbitrary fixed elements $\xi_{1}, \ldots, \xi_{\mu} \in \Xi$, $y_{1}, \ldots, y_{j-1}, y_{j+1}, \ldots, y_{m} \in Y$ there exists an element $\omega_{j} \in \Omega$ such that 
$\omega_{j} y=D\left(\begin{array}{ccc}\xi_{1}, & \ldots, & \xi_{\mu} \\ y_{1}, \ldots, & y_{j-1}, y, y_{j+1}, \ldots, & y_{m}\end{array}\right)$ for every $y \in Y(j=1, \ldots, m) . L_{\mu, m}(\Xi, Y)$

is identified with the set of all $(\Omega, X)$ - functionals on $\Xi^{\mu} \times Y^{m}$. A bilinear $(\Omega, X)$ - functional $D$ on $\Xi \times Y$ is said to be an operator on $\Xi \times Y$ and $\xi D y$ stands for its value at $(\xi, y)$. We denote by $o p(\Xi \rightarrow \Omega, Y \rightarrow X)$ the set of all $(\Omega, X)$ - operators on $\Xi \times Y$. Each $D \in o p(\Xi \rightarrow \Omega, Y \rightarrow X)$ can be simultaneously interpreted as a linear mapping $D: \Xi \rightarrow \Omega$ and as a linear mapping $D: Y \rightarrow X$. Thus $\xi D y=(\xi D) y=\xi(D y)$ for $(\xi, y) \in \Xi \times Y$. The operator $x_{0} \cdot \omega_{0} \in o p(\Xi \rightarrow \Omega, Y \rightarrow X), x_{0} \in X, \omega_{0} \in \Omega$ being fixed non-zero elements, defined by $\xi\left(x_{0} \cdot \omega_{0}\right) y=\xi x_{0} \cdot \omega_{0} y$ for $(\xi, y) \in \Xi \times Y$, is called one-dimensional.

For $A \in o p(\Omega \rightarrow \Xi, X \rightarrow Y)$ let $N(A)=\{x \in X: A x=0\}, R(A)=\{A x: x \in X\}$, $\mathfrak{n}(A)=\{\omega \in \Omega: \omega A=0\}, \boldsymbol{R}(A)=\{\omega A: \omega \in \Omega\} . A$ is said to be a Fredholm operator on $\Omega \times X \quad$ of order $r(A)=\min \left\{n^{\prime}, m^{\prime}\right\} \quad$ and index $d(A)=n^{\prime}-m^{\prime}, \quad$ if $\operatorname{dim} N(A)=n^{\prime}<\infty, \operatorname{dim} n(A)=m^{\prime}<\infty, R(A)=\eta(A)^{\perp}$ and $\mathbb{R}(A)=N(A)^{\perp}[5,21]$. An operator $B \in o p(\Xi \rightarrow \Omega, Y \rightarrow X)$ satisfying identities $A B A=A, B A B=B$ is called a reflexive generalized inverse of $A$ [22]. A sequence $\left(D_{n}\right)_{n \in N_{0}}$ is said to be a determinant system for $A$ if $D_{n} \in b_{s s} \mu_{\mu_{n}, m_{n}}(\Xi, Y) \cap L_{\mu_{n}, m_{n}}(\Xi, Y)$, with $\mu_{n}, m_{n} \in N_{0}$, $\mu_{n}=\mu_{0}+n, \quad m_{n}=m_{0}+n, \min \left(\mu_{0}, m_{0}\right)=0$, and the generalized Laplace expansion formulas hold

$$
\begin{gathered}
D_{n+1}\left(\begin{array}{ccc}
\xi_{0}, & \ldots, & \xi_{\mu_{n}} \\
A x, & y_{1}, \ldots, & y_{m_{n}}
\end{array}\right) \\
D_{n+1}\left(\begin{array}{ccc}
\omega A, & \xi_{1}, \ldots, & \xi_{\mu_{n}} \\
y_{0}, & \ldots, & y_{m_{n}}
\end{array}\right)=\sum_{j=0}^{m_{n}}(-1)^{i} \xi_{i} x \cdot D_{n}\left(\begin{array}{ccc}
\xi_{0}, \ldots, & \xi_{i-1}, \xi_{i+1}, \ldots, & \xi_{\mu_{n}} \\
y_{1}, & \ldots, & y_{m_{n}}
\end{array}\right),
\end{gathered}
$$

where $x \in X, \omega \in \Omega, \xi_{i} \in \Xi, y_{j} \in Y, i=1, \ldots, \mu_{n}, j=1, \ldots, m_{n}$. The least $r \in N_{0}$, such that $D_{r} \neq 0$, and the difference $\mu_{0}-m_{0}$ are called the order and the index of $\left(D_{n}\right)_{n \in N_{0}}$, respectively.

As well-known $[3,5]$, an operator $A \in o p(\Omega \rightarrow \Xi, X \rightarrow Y)$ has a determinant system $\left(D_{n}\right)_{n \in N_{0}}$ if and only if $A$ is Fredholm; the orders (the indices) of $A$ and $\left(D_{n}\right)_{n \in N_{0}}$ are the same. Moreover, if $A$ is Fredholm, $B \in o p(\Xi \rightarrow \Omega, Y \rightarrow X)$ is its reflexive generalized inverse and $\left\{z_{1}, \ldots, z_{n^{\prime}}\right\},\left\{\varsigma_{1}, \ldots, \varsigma_{m^{\prime}}\right\} \quad\left(\min \left\{n^{\prime}, m^{\prime}\right\}=r\right)$ 
form complete systems of solutions of the homogenous equations $A x=0$ and $\omega A=0$, respectively, then $\left(D_{n}\right)_{n \in N_{0}}$ defined by the formula

$$
D_{n}\left(\begin{array}{cc}
\xi_{1}, \ldots, & \xi_{n+n^{\prime}-r} \\
y_{1}, \ldots, & y_{n+m^{\prime}-r}
\end{array}\right)=\left|\begin{array}{cccccc}
\xi_{1} B y_{1} & \ldots & \xi_{1} B y_{n+m^{\prime}-r} & \xi_{1} z_{1} & \ldots & \xi_{1} z_{n^{\prime}} \\
\vdots & & \vdots & \vdots & & \vdots \\
\xi_{n+n^{\prime}-r} B y_{1} & \ldots & \xi_{n+n^{\prime}-r} B y_{n+m^{\prime}-r} & \xi_{n+n^{\prime}-r} z_{1} & \ldots & \xi_{n+n^{\prime}-r} z_{n^{\prime}} \\
\varsigma_{1} y_{1} & \ldots & \varsigma_{1} y_{n+m^{\prime}-r} & 0 & \ldots & 0 \\
\vdots & & \vdots & \vdots & & \vdots \\
\varsigma_{m^{\prime}} y_{1} & \ldots & \varsigma_{m^{\prime}} y_{n+m^{\prime}-r} & 0 & \ldots & 0
\end{array}\right|
$$

for $\xi_{i} \in \Xi\left(i=1, \ldots, n+n^{\prime}-r\right), y_{j} \in Y\left(j=1, \ldots, n+m^{\prime}-r\right)$, is a determinant system for $A$.

\section{Main result}

In this section we examine Fredholm operators acting from one linear space into another one. We provide a construction of a determinant system for a product of two fixed Fredholm operators. For the sake of completeness, we start by quoting some auxiliary results concerning reflexive generalized inverses of Fredholm operators, which are necessary for the proof of the main theorem of the paper.

In what follows, $A_{1} \in o p(\Omega \rightarrow \Xi, X \rightarrow Y), A_{2} \in o p(\Lambda \rightarrow \Omega, Y \rightarrow Z)$ denote Fredholm operators of orders $r\left(A_{1}\right)=r^{\prime}=\min \left\{n^{\prime}, m^{\prime}\right\}, r\left(A_{2}\right)=r^{\prime \prime}=\min \left\{n^{\prime \prime}, m^{\prime \prime}\right\}$ and indices $d\left(A_{1}\right)=d^{\prime}=n^{\prime}-m^{\prime}, d\left(A_{2}\right)=d^{\prime \prime}=n^{\prime \prime}-m^{\prime \prime}$, respectively. Let $\left(x_{1}^{\prime}, \ldots, x_{n^{\prime}}^{\prime}\right)$, $\left(\omega_{1}^{\prime}, \ldots, \omega_{m^{\prime}}^{\prime}\right),\left(y_{1}^{\prime \prime}, \ldots, y_{n^{\prime \prime}}^{\prime \prime}\right)$ and $\left(\lambda_{1}^{\prime \prime}, \ldots, \lambda_{m^{\prime \prime}}^{\prime \prime}\right)$ be bases of $N\left(A_{1}\right), \mathfrak{n}\left(A_{1}\right), N\left(A_{2}\right)$ and $\mathfrak{n}\left(A_{2}\right)$, respectively. The following direct sum decompositions hold: $Y=R\left(A_{1}\right) \oplus Y^{\prime}, \quad \Omega=\mathbb{R}\left(A_{2}\right) \oplus \Omega^{\prime \prime}, Y^{\prime} \subseteq Y, \Omega^{\prime \prime} \subseteq \Omega$ being subspaces such as $\operatorname{dim} Y^{\prime}=m^{\prime}$ and $\operatorname{dim} \Omega^{\prime \prime}=n^{\prime \prime}$. Moreover, denoting $Y_{1}=N\left(A_{2}\right) \cap R\left(A_{1}\right)$, $\Omega_{1}=\mathfrak{n}\left(A_{1}\right) \cap \mathbb{R}\left(A_{2}\right)$, we also obtain $N\left(A_{2}\right)=Y_{1} \oplus Y_{2}, \quad \mathfrak{n}\left(A_{1}\right)=\Omega_{1} \oplus \Omega_{2}$, where $Y_{2}=N\left(A_{2}\right) \cap Y^{\prime}, \Omega_{2}=\mathfrak{n}\left(A_{1}\right) \cap \Omega^{\prime \prime}$ and $\operatorname{dim} Y_{2}=\operatorname{dim} \Omega_{2}=t$. Let $\left(y_{1}^{\prime \prime}, \ldots, y_{n^{\prime \prime}-t}^{\prime \prime}\right)$, $\left(\omega_{1}^{\prime}, \ldots, \omega_{m^{\prime}-t}^{\prime}\right)$ be bases of subspaces $Y_{1}, \Omega_{1}$, respectively, and $Y_{2}=\operatorname{span}\left(y_{1}, \ldots, y_{t}\right)$, $\Omega_{2}=\operatorname{span}\left(\omega_{1}, \ldots, \omega_{t}\right)$, where $\omega_{i} y_{j}=\delta_{i j} \quad(i, j=1, \ldots, t), \delta_{i j}$ being the Kronecker symbol. Furthermore, $Y^{\prime}=Y_{2} \oplus Y_{3}, \Omega^{\prime \prime}=\Omega_{2} \oplus \Omega_{3}, Y_{3} \subseteq Y, \Omega_{3} \subseteq \Omega$ being subspaces of dimensions $m^{\prime}-t, n^{\prime \prime}-t$, respectively.

Under the above given assumptions we recall [20] the following two results. 
Lemma 3.1. If $B_{1} \in o p(\Xi \rightarrow \Omega, Y \rightarrow X), \quad B_{2} \in o p(\Omega \rightarrow \Lambda, Z \rightarrow Y)$ are arbitrary reflexive generalized inverses of Fredholm operators $A_{1}, A_{2}$, respectively, then $\left(x_{1}^{\prime}, \ldots, x_{n^{\prime}}^{\prime}, B_{1} y_{1}^{\prime \prime}, \ldots, B_{1} y_{n^{\prime \prime}-t}^{\prime \prime}\right)$ and $\left(\lambda_{1}^{\prime \prime}, \ldots, \lambda_{m^{\prime \prime}}^{\prime \prime}, \omega_{1}^{\prime} B_{2}, \ldots, \omega_{m^{\prime}-t}^{\prime} B_{2}\right)$ are bases of $N\left(A_{2} A_{1}\right)$ and $n\left(A_{2} A_{1}\right)$, respectively.

Lemma 3.2. We assume that:

(i) $\left(D_{n}^{(1)}\right)_{n \in N_{0}},\left(D_{n}^{(2)}\right)_{n \in N_{0}}$ are fixed determinant systems for Fredholm operators $A_{1}, A_{2}$, respectively;

(ii) $\xi_{1}^{\prime}, \ldots, \xi_{n^{\prime}}^{\prime} \in \Xi \quad y_{1}^{\prime}, \ldots, y_{m^{\prime}-t}^{\prime} \in Y_{3}$ are such elements that:

$$
\delta^{\prime}=D_{r^{\prime}}^{(1)}\left(\begin{array}{ccc}
\xi_{1}^{\prime}, & \ldots, & \xi_{n^{\prime}}^{\prime} \\
y_{1}^{\prime}, \ldots, & y_{m^{\prime}-t}^{\prime}, y_{1}, \ldots, & y_{t}
\end{array}\right) \neq 0 ;
$$

(iii) $z_{1}^{\prime \prime}, \ldots, z_{m^{\prime \prime}}^{\prime \prime} \in Z, \omega_{1}^{\prime \prime}, \ldots, \omega_{n^{\prime \prime}-t}^{\prime \prime} \in \Omega_{3}$ are such elements that:

$$
\delta^{\prime \prime}=D_{r^{\prime \prime}}^{(2)}\left(\begin{array}{ccc}
\omega_{1}^{\prime \prime}, \ldots, & \omega_{n^{\prime \prime}-t}^{\prime \prime}, \omega_{1}, \ldots, & \omega_{t} \\
z_{1}^{\prime \prime}, & \ldots, & z_{m^{\prime \prime}}^{\prime \prime}
\end{array}\right) \neq 0 ;
$$

(iv) $B_{1} \in o p(\Xi \rightarrow \Omega, Y \rightarrow X)$ is a reflexive generalized inverse of $A_{1}$ defined by the formula

$$
\xi B_{1} y=\frac{1}{\delta^{\prime}} D_{r^{\prime}+1}^{(1)}\left(\begin{array}{cccc}
\xi, & \xi_{1}^{\prime}, & \ldots, & \xi_{n^{\prime}}^{\prime} \\
y, & y_{1}^{\prime}, \ldots, & y_{m^{\prime}-t}^{\prime}, y_{1}, \ldots, & y_{t}
\end{array}\right) \text { for }(\xi, y) \in \Xi \times Y ;
$$

(v) $B_{2} \in o p(\Omega \rightarrow \Lambda, Z \rightarrow Y)$ is a reflexive generalized inverse of $A_{2}$ defined by the formula

$$
\omega B_{2} z=\frac{1}{\delta^{\prime \prime}} D_{r^{\prime \prime}+1}^{(2)}\left(\begin{array}{cccc}
\omega, & \omega_{1}^{\prime \prime}, \ldots, & \omega_{n^{\prime \prime}-t}^{\prime \prime}, \omega_{1}, \ldots, & \omega_{t} \\
z, & z_{1}^{\prime \prime}, & \ldots, & z_{m^{\prime \prime}}^{\prime \prime}
\end{array}\right) \text { for }(\omega, z) \in \Omega \times Z .
$$

Then the operator $B_{1} B_{2} \in o p(\Xi \rightarrow \Lambda, Z \rightarrow X)$ is a reflexive generalized inverse of $A_{2} A_{1} \in o p(\Lambda \rightarrow \Xi, X \rightarrow Z)$.

The following lemma plays an essential role in the sequel. It describes the connection between two arbitrary reflexive generalized inverses of a fixed Fredholm operator.

Lemma 3.3. If $B, C \in o p(\Xi \rightarrow \Omega, Y \rightarrow X)$ are reflexive generalized inverses of a Fredholm operator $A \in o p(\Omega \rightarrow \Xi, X \rightarrow Y)$ and $\left(\widetilde{x}_{1}, \ldots, \widetilde{x}_{n}\right),\left(\widetilde{\omega}_{1}, \ldots, \widetilde{\omega}_{m}\right)$ are bases of $N(A), \quad \eta(A)$, respectively, then there exist elements $\eta_{i} \in \mathbb{R}(B)$ $(i=1, \ldots, n), u_{j} \in R(B)(j=1, \ldots, m)$ such that 


$$
C=B+\sum_{i=1}^{n} \widetilde{x}_{i} \cdot \eta_{i}+\sum_{j=1}^{m} u_{j} \cdot \widetilde{\omega}_{j}+\sum_{i=1}^{n} \sum_{j=1}^{m}\left(\eta_{i} A u_{j}\right) \widetilde{x}_{i} \cdot \widetilde{\omega}_{j}
$$

Proof. By the relationship between $A$ and its reflexive generalized inverse $B$ [5], there exist elements $\widetilde{\xi}_{1}^{\prime}, \ldots, \widetilde{\xi}_{n}^{\prime} \in \Xi, \quad \widetilde{y}_{1}^{\prime}, \ldots, \widetilde{y}_{m}^{\prime} \in Y \quad$ such that $\widetilde{\xi}_{i}^{\prime} \widetilde{x}_{j}=\delta_{i j}$ $(i, j=1, \ldots, n), \widetilde{\omega}_{i} \widetilde{y}_{j}^{\prime}=\delta_{i j}(i, j=1, \ldots, m)$ and the following identities hold:

$$
B A=I-\sum_{i=1}^{n} \widetilde{x}_{i} \cdot \widetilde{\xi}_{i}^{\prime}, \quad A B=J-\sum_{j=1}^{m} \widetilde{y}_{j}^{\prime} \cdot \widetilde{\omega}_{j}
$$

Similarly, in view of the relationship between $A$ and $C$,

$$
C A=I-\sum_{i=1}^{n} \widetilde{x}_{i} \cdot \widetilde{\xi}_{i}^{\prime \prime}, A C=J-\sum_{j=1}^{m} \widetilde{y}_{j}^{\prime \prime} \cdot \widetilde{\omega}_{j},
$$

where $\widetilde{\xi}_{1}^{\prime \prime}, \ldots, \widetilde{\xi}_{n}^{\prime \prime} \in \Xi, \widetilde{y}_{1}^{\prime \prime}, \ldots, \widetilde{y}_{m}^{\prime \prime} \in Y$ are elements satisfying conditions: $\widetilde{\xi}_{i}^{\prime \prime} \widetilde{x}_{j}=\delta_{i j}$ $(i, j=1, \ldots, n), \quad \widetilde{\omega}_{i} \widetilde{y}_{j}^{\prime \prime}=\delta_{i j}(i, j=1, \ldots, m)$. By (5) and (6), bearing in mind that $\widetilde{\xi}_{i}^{\prime} B=0 \quad(i=1, \ldots, n), \quad C A B-B A B=\left(\sum_{i=1}^{n} \widetilde{x}_{i} \cdot \widetilde{\xi}_{i}^{\prime}-\sum_{i=1}^{n} \widetilde{x}_{i} \cdot \widetilde{\xi}_{i}^{\prime \prime}\right) B=-\sum_{i=1}^{n} \widetilde{x}_{i} \cdot \widetilde{\xi}_{i}^{\prime \prime} B$. Consequently, $C\left(J-\sum_{j=1}^{m} \widetilde{y}_{j}^{\prime} \cdot \widetilde{\omega}_{j}\right)-B=-\sum_{i=1}^{n} \widetilde{x}_{i} \cdot \widetilde{\xi}_{i}^{\prime \prime} B$, which implies

$$
C=B-\sum_{i=1}^{n} \widetilde{x}_{i} \cdot \widetilde{\xi}_{i}^{\prime \prime} B+\sum_{j=1}^{m} C \widetilde{y}_{j}^{\prime} \cdot \widetilde{\omega}_{j} .
$$

It follows from (7) that

$$
C A C=\left(B A-\sum_{i=1}^{n} \widetilde{x}_{i} \cdot \widetilde{\xi}_{i}^{\prime \prime} B A+\sum_{j=1}^{m} C \widetilde{y}_{j}^{\prime} \cdot \widetilde{\omega}_{j} A\right)\left(B-\sum_{i=1}^{n} \widetilde{x}_{i} \cdot \widetilde{\xi}_{i}^{\prime \prime} B+\sum_{j=1}^{m} C \widetilde{y}_{j}^{\prime} \cdot \widetilde{\omega}_{j}\right) .
$$

Since $\widetilde{\omega}_{i} \in \mathfrak{n}(A)(i=1, \ldots, n)$, we transform the right-hand side of $(8)$ into the form

$$
\begin{gathered}
B A B-\sum_{i=1}^{n} \widetilde{x}_{i} \cdot \widetilde{\xi}_{i}^{\prime \prime} B A B-\sum_{i=1}^{n} B A \widetilde{x}_{i} \cdot \widetilde{\xi}_{i}^{\prime \prime} B+\sum_{i=1}^{n} \sum_{k=1}^{n}\left(\widetilde{\xi}_{i}^{\prime \prime} B A \widetilde{x}_{k}\right) \widetilde{x}_{i} \cdot \widetilde{\xi}_{k}^{\prime \prime} B+ \\
+\sum_{j=1}^{m} B A C \widetilde{y}_{j}^{\prime} \cdot \widetilde{\omega}_{j}-\sum_{i=1}^{n} \sum_{j=1}^{m}\left(\widetilde{\xi}_{i}^{\prime \prime} B A C \widetilde{y}_{j}^{\prime}\right) \widetilde{x}_{i} \cdot \widetilde{\omega}_{j} \cdot
\end{gathered}
$$


Furthermore, remembering that $\widetilde{x}_{i} \in N(A)(i=1, \ldots, n)$, we express (9) by

$$
B A B-\sum_{i=1}^{n} \widetilde{x}_{i} \cdot \widetilde{\xi}_{i}^{\prime \prime} B A B+\sum_{j=1}^{m} B A C \widetilde{y}_{j}^{\prime} \cdot \widetilde{\omega}_{j}-\sum_{i=1}^{n} \sum_{j=1}^{m}\left(\widetilde{\xi}_{i}^{\prime \prime} B A C \widetilde{y}_{j}^{\prime}\right) \widetilde{x}_{i} \cdot \widetilde{\omega}_{j} \cdot
$$

Hence, the identities $B A B=B, C A C=C$, combined with (8) and (10), lead to

$$
C=B-\sum_{i=1}^{n} \widetilde{x}_{i} \cdot \widetilde{\xi}_{i}^{\prime \prime} B+\sum_{j=1}^{m} B A C \widetilde{y}_{j}^{\prime} \cdot \widetilde{\omega}_{j}-\sum_{i=1}^{n} \sum_{j=1}^{m}\left[\left(\widetilde{\xi}_{i}^{\prime \prime} B\right) A\left(B A C \widetilde{y}_{j}^{\prime}\right)\right] \widetilde{x}_{i} \cdot \widetilde{\omega}_{j}
$$

Finally, by putting $\eta_{i}=-\widetilde{\xi}_{i}^{\prime \prime} B \quad(i=1, \ldots, n)$ and $u_{j}=B A C \widetilde{y}_{j}^{\prime} \quad(j=1, \ldots, m)$ in (11), we arrive at (4), which is the required result.

Having established Lemmas 3.1-3.3, we are now in a position to state and prove the main result of the paper.

Theorem 3.4. Let $\left(D_{m}^{(1)^{n}}\right)_{n, m \in N_{0}}\left(n-m=d^{\prime}\right), \quad\left(D_{m}^{(2)_{m}^{n}}\right)_{n, m \in N_{0}}\left(n-m=d^{\prime \prime}\right)$ be fixed determinant systems for Fredholm operators $A_{1}, A_{2}$, respectively. If $C_{1} \in o p(\Xi \rightarrow \Omega, Y \rightarrow X), C_{2} \in o p(\Omega \rightarrow \Lambda, Z \rightarrow Y)$ are arbitrary reflexive generalized inverses of $A_{1}, A_{2}$, respectively, then the sequence $\left(D_{m}^{n}\right)$ $\left(n \geq \max \left\{d^{\prime}+d^{\prime \prime}, 0\right\}, \quad n-m=d^{\prime}+d^{\prime \prime}\right)$ defined by the formula:

$$
\begin{aligned}
& D_{m}^{n}\left(\begin{array}{cc}
\xi_{1}, \ldots, & \xi_{n} \\
z_{1}, \ldots, & z_{m}
\end{array}\right)=\sum_{p, q} \operatorname{sgn} p \operatorname{sgn} q D_{m-m^{\prime}+t}^{(2)^{n-n^{\prime}+t}}\left(\begin{array}{ccc}
\xi_{p_{1}} C_{1}, \ldots, & \xi_{p_{n-n^{\prime}}} C_{1}, \omega_{1}, \ldots, & \omega_{t} \\
z_{q_{1}}, & \ldots, & z_{q_{m-m^{\prime}+t}}
\end{array}\right) \times \\
& \times D^{(1)_{m^{\prime}}^{n^{\prime}}}\left(\begin{array}{ccc}
\xi_{p_{n-n^{\prime}+1}}, & \ldots, & \xi_{p_{n}} \\
C_{2} z_{q_{m-m^{\prime}+t+1}}, \ldots, & C_{2} z_{q_{m}}, y_{1}, \ldots, & y_{t}
\end{array}\right)
\end{aligned}
$$

for $\xi_{i} \in \Xi, z_{j} \in Z \quad(i=1, \ldots, n, j=1, \ldots, m)$, where $p=\left(p_{1}, \ldots, p_{n}\right), q=\left(q_{1}, \ldots, q_{m}\right)$ are arbitrary permutations of integers $1, \ldots, n$ and $1, \ldots, m$, respectively, such that

$$
p_{1}<\ldots<p_{n-n^{\prime}}, \quad p_{n-n^{\prime}+1}<\ldots<p_{n}, \quad q_{1}<\ldots<q_{m-m^{\prime}+t}, \quad q_{m-m^{\prime}+t+1}<\ldots<q_{m},
$$

is a determinant system for $A_{2} A_{1} \in o p(\Lambda \rightarrow \Xi, X \rightarrow Z)$.

Proof. Let $B_{1}, B_{2}$ be reflexive generalized inverses of $A_{1}, A_{2}$, respectively, defined by formulas (2), (3). According to Lemma 3.2, $B_{1} B_{2}$ is a reflexive generalized inverse of operator $A_{2} A_{1}$. It follows from (1), in view of Lemma 3.1, that the sequence $\left(\widetilde{D}_{m}^{n}\right)$ defined by 


$$
\begin{gathered}
\widetilde{D}_{m}^{n}\left(\begin{array}{cccccccc}
\xi_{1}, \ldots, & \xi_{n} \\
z_{1}, \ldots, & z_{m}
\end{array}\right)= \\
=\left|\begin{array}{ccccccccc}
\xi_{1} B_{1} B_{2} z_{1} & \ldots & \xi_{1} B_{1} B_{2} z_{m} & \xi_{1} B_{1} y_{1}^{\prime \prime} & \ldots & \xi_{1} B_{1} y_{n^{\prime \prime}-t}^{\prime \prime} & \xi_{1} x_{1}^{\prime} & \ldots & \xi_{1} x_{n^{\prime}}^{\prime} \\
\vdots & & \vdots & \vdots & & \vdots & \vdots & & \vdots \\
\xi_{n} B_{1} B_{2} z_{1} & \ldots & \xi_{n} B_{1} B_{2} z_{m} & \xi_{n} B_{1} y_{1}^{\prime \prime} & \ldots & \xi_{n} B_{1} y_{n^{\prime \prime}-t}^{\prime \prime} & \xi_{n} x_{1}^{\prime} & \ldots & \xi_{n} x_{n^{\prime}}^{\prime} \\
\lambda_{1}^{\prime \prime} z_{1} & \ldots & \lambda_{1}^{\prime \prime} z_{m} & 0 & \ldots & 0 & 0 & \ldots & 0 \\
\vdots & & \vdots & \vdots & & \vdots & \vdots & & \vdots \\
\lambda_{m^{\prime \prime}}^{\prime \prime} z_{1} & \ldots & \lambda_{m^{\prime \prime}}^{\prime \prime} z_{m} & 0 & \ldots & 0 & 0 & \ldots & 0 \\
\omega_{1}^{\prime} B_{2} z_{1} & \ldots & \omega_{1}^{\prime} B_{2} z_{m} & 0 & \ldots & 0 & 0 & \ldots & 0 \\
\vdots & & \vdots & \vdots & & \vdots & \vdots & & \vdots \\
\omega_{m^{\prime}-t}^{\prime} B_{2} z_{1} & \ldots & \omega_{m^{\prime}-t}^{\prime} B_{2} z_{m} & 0 & \ldots & 0 & 0 & \ldots & 0
\end{array}\right| \\
\left(n \geq \max \left\{d^{\prime}+d^{\prime \prime}, 0\right\}, n-m=d^{\prime}+d^{\prime \prime}\right) \text { for } \xi_{i} \in \Xi, z_{j} \in Z(i=1, \ldots, n, j=1, \ldots, m),
\end{gathered}
$$
is a determinant system for $A_{2} A_{1}$. The order and the index of $\left(\widetilde{D}_{m}^{n}\right)$ are equal to $r=\min \left\{n^{\prime}+n^{\prime \prime}-t, m^{\prime}+m^{\prime \prime}-t\right\}$ and $d=d^{\prime}+d^{\prime \prime}$, respectively. Let $\widetilde{C}_{2}$ denote the reflexive generalized inverse of $A_{2}$ expressed by

$$
\widetilde{C}_{2}=B_{2}+\sum_{i=1}^{n^{\prime \prime}-t} y_{i}^{\prime \prime} \cdot \eta_{i}^{\prime \prime}+\sum_{j=1}^{m^{\prime \prime}} u_{j}^{\prime \prime} \cdot \lambda_{j}^{\prime \prime}+\sum_{i=1}^{n^{\prime \prime}-t} \sum_{j=1}^{m^{\prime \prime}}\left(\eta_{i}^{\prime \prime} A_{2} u_{j}^{\prime \prime}\right) y_{i}^{\prime \prime} \cdot \lambda_{j}^{\prime \prime}
$$

where $\eta_{i}^{\prime \prime} \in \mathbb{R}\left(B_{2}\right)\left(i=1, \ldots, n^{\prime \prime}-t\right), u_{j}^{\prime \prime} \in R\left(B_{2}\right)\left(j=1, \ldots, m^{\prime \prime}\right)$. Since $\omega_{k} \in R\left(A_{1}\right)^{\perp}$ and $\omega_{k} B_{2}=0 \quad(k=1, \ldots, t), \quad \omega_{k} \widetilde{C}_{2}=0$. Let $C_{1}$ be an arbitrary fixed reflexive generalized inverse of $A_{1}$. Hence, by Lemma 3.3,

$$
C_{1}=B_{1}+\sum_{i=1}^{n^{\prime}} x_{i}^{\prime} \cdot \eta_{i}^{\prime}+\sum_{j=1}^{m^{\prime}} u_{j}^{\prime} \cdot \omega_{j}^{\prime}+\sum_{i=1}^{n^{\prime}} \sum_{j=1}^{m^{\prime}}\left(\eta_{i}^{\prime} A_{1} u_{j}^{\prime}\right) x_{i}^{\prime} \cdot \omega_{j}^{\prime},
$$

for some $\eta_{i}^{\prime} \in \mathbb{R}\left(B_{1}\right)\left(i=1, \ldots, n^{\prime}\right), u_{j}^{\prime} \in R\left(B_{1}\right)\left(j=1, \ldots, m^{\prime}\right)$. Assume $y_{n^{\prime \prime}-t+i}^{\prime \prime}=y_{i}$, $\omega_{m^{\prime}-t+i}^{\prime}=\omega_{i}(i=1, \ldots, t)$. The orthogonality of $y_{1}^{\prime \prime}, \ldots, y_{n^{\prime \prime}-t}^{\prime \prime}$ to all $\omega_{1}^{\prime}, \ldots, \omega_{m^{\prime}}^{\prime}$ and the orthogonality of $\omega_{1}^{\prime}, \ldots, \omega_{m^{\prime}-t}^{\prime}$ to all $y_{1}^{\prime \prime}, \ldots, y_{n^{\prime \prime}}^{\prime \prime}$, combined with (14), (15), lead to $C_{1} \widetilde{C}_{2}=B_{1} B_{2}+S$, where $S \in o p(\Xi \rightarrow \Lambda, Z \rightarrow X)$ is a finitely dimensional operator of the form 


$$
S=\sum_{i=1}^{n^{\prime \prime}-t} B_{1} y_{i}^{\prime \prime} \cdot \lambda_{i}+\sum_{i=1}^{n^{\prime}} x_{i}^{\prime} \cdot \lambda_{n^{\prime \prime}-t+i}+\sum_{i=1}^{m^{\prime}-t} x_{i} \cdot \omega_{i}^{\prime} B_{2}+\sum_{i=1}^{m^{\prime \prime}} x_{m^{\prime}-t+i} \cdot \lambda_{i}^{\prime \prime},
$$

for some $\lambda_{i} \in \Lambda\left(i=1, \ldots, n^{\prime}+n^{\prime \prime}-t\right), \quad x_{j} \in X\left(j=1, \ldots, m^{\prime}+m^{\prime \prime}-t\right)$. Moreover, by virtue of (15), for any $\xi_{i} \in \Xi(i=1, \ldots, n)$

$$
\xi_{i} C_{1} y_{j}^{\prime \prime}=\xi_{i}\left(B_{1}+\sum_{k=1}^{n^{\prime}} x_{k}^{\prime} \cdot \eta_{k}^{\prime}\right) y_{j}^{\prime \prime} \quad\left(j=1, \ldots, n^{\prime \prime}-t\right) .
$$

Similarly, by (14), for any $z_{j} \in Z(j=1, \ldots, m)$

$$
\omega_{i}^{\prime} \widetilde{C}_{2} z_{j}=\omega_{i}^{\prime}\left(B_{2}+\sum_{k=1}^{m^{\prime \prime}} u_{k}^{\prime \prime} \cdot \lambda_{k}^{\prime \prime}\right) z_{j} \quad\left(i=1, \ldots, m^{\prime}-t\right) .
$$

Replacing $B_{1}$ by $C_{1}$ and $B_{2}$ by $\widetilde{C}_{2}$ in (13), we conclude that the right-hand side of (13) remains unchanged. Furthermore, applying the formula for the generalized expansion of a determinant, we transform the determinant in (13) into the sum

$$
\sum_{p} \operatorname{sgn} p\left|\begin{array}{cccccc}
\lambda_{1}^{\prime \prime} z_{1} & \ldots & \lambda_{1}^{\prime \prime} z_{m} & 0 & \ldots & 0 \\
\vdots & & \vdots & \vdots & & \vdots \\
\lambda_{m^{\prime \prime}}^{\prime \prime} z_{1} & \ldots & \lambda_{m^{\prime \prime}}^{\prime \prime} z_{m} & 0 & \ldots & 0 \\
\omega_{1}^{\prime} \tilde{C}_{2} z_{1} & \ldots & \omega_{1}^{\prime} \tilde{C}_{2} z_{m} & 0 & \ldots & 0 \\
\vdots & & \vdots & \vdots & & \vdots \\
\omega_{m^{\prime}-t}^{\prime} \tilde{C}_{2} z_{1} & \ldots & \omega_{m^{\prime}-t}^{\prime} \tilde{C}_{2} z_{m} & 0 & \ldots & 0 \\
\xi_{p_{1}} C_{1} \tilde{C}_{2} z_{1} & \ldots & \xi_{p_{1}} C_{1} \tilde{C}_{2} z_{m} & \xi_{p_{1}} C_{1} y_{1}^{\prime \prime} & \ldots & \xi_{p_{1}} C_{1} y_{n^{\prime \prime}-t}^{\prime \prime} \\
\vdots & & \vdots & \vdots & & \vdots \\
\xi_{p_{n-n^{\prime}}} C_{1} \tilde{C}_{2} z_{1} & \ldots & \xi_{p_{n-n^{\prime}}} C_{1} \tilde{C}_{2} z_{m} & \xi_{p_{n-n^{\prime}}} C_{1} y_{1}^{\prime \prime} & \ldots & \xi_{p_{n-n^{\prime}}} C_{1} y_{n^{\prime \prime}-t}^{\prime \prime}
\end{array}\right|\left|\begin{array}{cccc}
\xi_{p_{n-n^{\prime \prime}+1}} x_{1}^{\prime} & \ldots & \xi_{p_{n-n^{\prime}+1}} x_{n^{\prime}}^{\prime} \\
\vdots & & \vdots \\
\xi_{p_{n}} x_{1}^{\prime} & \ldots & \xi_{p_{n}} x_{n^{\prime}}^{\prime}
\end{array}\right|
$$

multiplied by $(-1)^{n\left(m^{\prime}+m^{\prime \prime}-t\right)}$, where $p=\left(p_{1}, \ldots, p_{n}\right)$ is a permutation of integers $1, \ldots, n$ fulfilling the condition $p_{1}<\ldots<p_{n-n^{\prime}}, \quad p_{n-n^{\prime}+1}<\ldots<p_{n}$. Moreover, denoting by $q$ any permutation of integers $1, \ldots, m$ such that $q_{1}<\ldots<q_{m-m^{\prime}+t}, q_{m-m^{\prime}+t+1}<$ $\ldots<q_{m}$ and making use of well-known properties of classical determinants, in view of (16), the right-hand side of (13) is equal, up to a sign, to the sum 


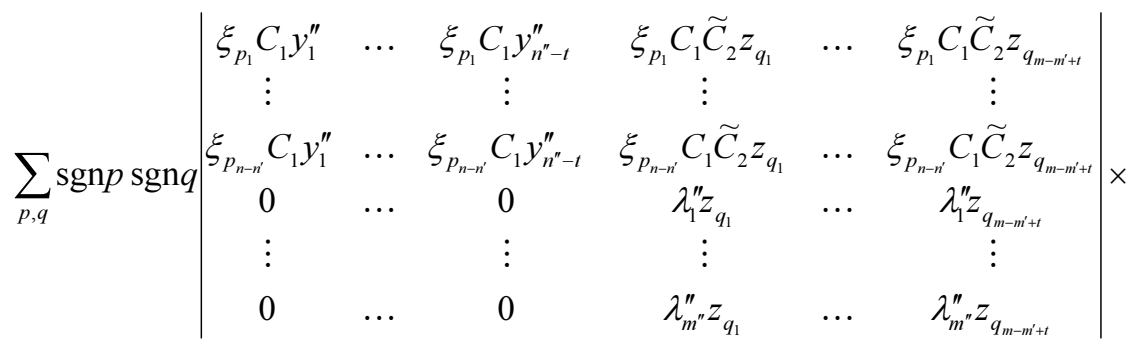

$$
\begin{aligned}
& \times\left|\begin{array}{ccc|ccc}
\omega_{1}^{\prime} \widetilde{C}_{2} z_{q_{m-n^{\prime}+t+1}} & \ldots & \omega_{1}^{\prime} \widetilde{C}_{2} z_{q_{m}} & \xi_{p_{n-n^{\prime}+1}} x_{1}^{\prime} & \ldots & \xi_{p_{n-n^{\prime}+1}} x_{n^{\prime}}^{\prime} \\
\vdots & & \vdots & \vdots & & \vdots \\
\omega_{m^{\prime}-t}^{\prime} \widetilde{C}_{2} z_{q_{m-n n^{\prime}+t+1}} & \ldots & \omega_{m^{\prime}-t}^{\prime} \widetilde{C}_{2} z_{q_{m}} & \xi_{p_{n}} x_{1}^{\prime} & \ldots & \xi_{p_{n}} x_{n^{\prime}}^{\prime}
\end{array}\right| .
\end{aligned}
$$

Next, taking into account the identities $\omega_{i} y_{j}^{\prime \prime}=0 \quad\left(i=1, \ldots, t, j=1, \ldots, n^{\prime \prime}-t\right)$, $\omega_{i}^{\prime} y_{j}=0 \quad\left(i=1, \ldots, m^{\prime}-t, j=1, \ldots, t\right), \quad \omega_{i} \widetilde{C}_{2}=0(i=1, \ldots, t) \quad$ and $\left.\operatorname{det} \mid \omega_{i} y_{j}\right\rfloor_{1 \leq i, j \leq t}=$ $=\operatorname{det}\left[\delta_{i j}\right\rfloor_{1 \leq i, j \leq t}=1$, the sum (17) can be expressed (up to a sign) by

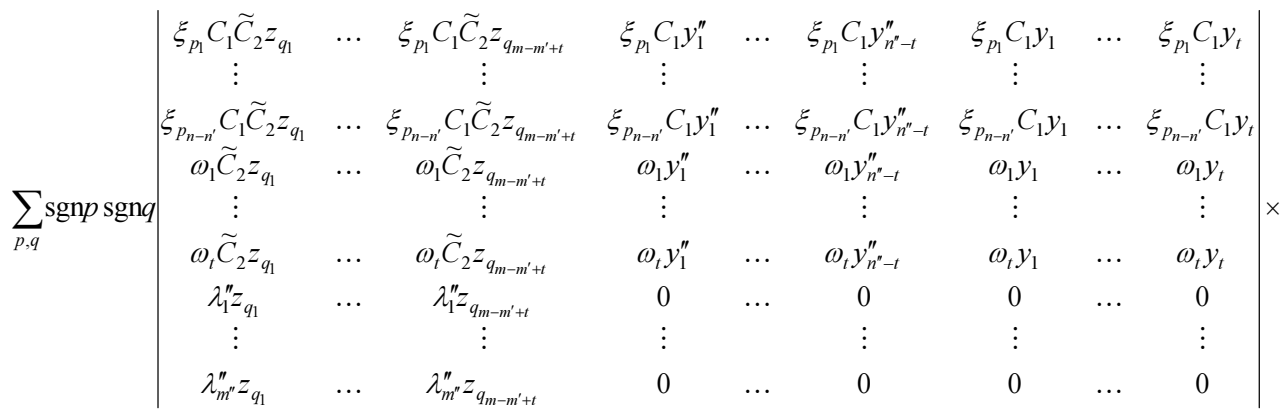

$$
\begin{aligned}
& \times\left|\begin{array}{cccccc}
\omega_{1}^{\prime} \widetilde{C}_{2} z_{q_{m-m^{\prime}+t+1}} & \ldots & \omega_{1}^{\prime} \widetilde{C}_{2} z_{q_{m}} & \omega_{1}^{\prime} y_{1} & \ldots & \omega_{1}^{\prime} y_{t} \\
\vdots & \vdots & \vdots & & \vdots \\
\omega_{m^{\prime}-t}^{\prime} \widetilde{C}_{2} z_{q_{m-n n^{\prime}+t+1}} & \ldots & \omega_{m^{\prime}-t}^{\prime} \widetilde{C}_{2} z_{q_{m}} & \omega_{m^{\prime}-t} y_{1} & \ldots & \omega_{m^{\prime}-t}^{\prime} y_{t} \\
\omega_{1} \widetilde{C}_{2} z_{q_{m-m^{\prime}+t+1}} & \ldots & \omega_{1} \widetilde{C}_{2} z_{q_{m}} & \omega_{1} y_{1} & \ldots & \omega_{1} y_{t} \\
\vdots & & \vdots & \vdots & & \vdots \\
\omega_{t} \widetilde{C}_{2} z_{q_{m-m^{\prime}+t+1}} & \ldots & \omega_{t} \widetilde{C}_{2} z_{q_{m}} & \omega_{t} y_{1} & \ldots & \omega_{t} y_{t}
\end{array}\right| \begin{array}{cccc} 
& & & \\
\vdots & x_{1}^{\prime} & \ldots & \xi_{p_{n-n^{\prime}+1}+1} x_{n^{\prime}}^{\prime} \\
\xi_{p_{n}} x_{1}^{\prime} & \ldots & \xi_{p_{n}} x_{n^{\prime}}^{\prime}
\end{array} \mid .
\end{aligned}
$$

It follows from (18), bearing in mind the definition of $\left(D_{m}^{(2)^{n}}\right)_{n, m \in N_{0}}$ and relying on properties of partitioned matrices, that the value $\widetilde{D}_{m}^{n}\left(\begin{array}{cc}\xi_{1}, \ldots, & \xi_{n} \\ z_{1}, \ldots, & z_{m}\end{array}\right)$ is equal, up to a factor of 1 or -1 , to 


$$
\begin{aligned}
& \sum_{p, q} \operatorname{sgn} p \operatorname{sgn} q D^{(2)_{m-m^{\prime}+t}^{n-n^{\prime}+t}}\left(\begin{array}{ccc}
\xi_{p_{1}} C_{1}, \ldots, & \xi_{p_{n-n^{\prime}}} C_{1}, \omega_{1}, \ldots, & \omega_{t} \\
z_{q_{1}}, & \ldots, & z_{q_{m-m^{\prime}+t}}
\end{array}\right) \times \\
& \times\left|\begin{array}{ccccccccc}
\xi_{p_{n-n^{\prime}+1}} C_{1} \widetilde{C}_{2} z_{q_{m-m^{\prime}+t+1}} & \ldots & \xi_{p_{n-n^{\prime}+1}} C_{1} \widetilde{C}_{2} z_{q_{m}} & \xi_{p_{n-n^{\prime}+1}} C_{1} y_{1} & \ldots & \xi_{p_{n-n^{\prime}+1}} C_{1} y_{t} & \xi_{p_{n-n^{\prime}+1}} x_{1}^{\prime} & \ldots & \xi_{p_{n-n^{\prime}+1}} x_{n^{\prime}}^{\prime} \\
\vdots & & \vdots & \vdots & & \vdots & \vdots & & \vdots \\
\xi_{p_{n}} C_{1} \widetilde{C}_{2} z_{q_{m-m^{\prime}+t+1}} & \ldots & \xi_{p_{n}} C_{1} \widetilde{C}_{2} z_{q_{m}} & \xi_{p_{n}} C_{1} y_{1} & \ldots & \xi_{p_{n}} C_{1} y_{t} & \xi_{p_{n}} x_{1}^{\prime} & \ldots & \xi_{p_{n}} x_{n^{\prime}}^{\prime} \\
\omega_{1}^{\prime} \widetilde{C}_{2} z_{q_{m-m^{\prime}+t+1}} & \ldots & \omega_{1}^{\prime} \widetilde{C}_{2} z_{q_{m}} & \omega_{1}^{\prime} y_{1} & \ldots & \omega_{1}^{\prime} y_{t} & 0 & \ldots & 0 \\
\vdots & & \vdots & \vdots & & \vdots & \vdots & & \vdots \\
\omega_{m^{\prime}-t}^{\prime} \widetilde{C}_{2} z_{q_{m-m^{\prime}+t+1}} & \ldots & \omega_{m^{\prime}-t}^{\prime} \widetilde{C}_{2} z_{q_{m}} & \omega_{m^{\prime}-t}^{\prime} y_{1} & \ldots & \omega_{m^{\prime}-t}^{\prime} y_{t} & 0 & \ldots & 0 \\
\omega_{1} \widetilde{C}_{2} z_{q_{m-m^{\prime}+t+1}} & \ldots & \omega_{1} \widetilde{C}_{2} z_{q_{m}} & \omega_{1} y_{1} & \ldots & \omega_{1} y_{t} & 0 & \ldots & 0 \\
\vdots & & \vdots & \vdots & & \vdots & \vdots & & \vdots \\
\omega_{t} \widetilde{C}_{2} z_{q_{m-m^{\prime}+t+1}} & \ldots & \omega_{t} \widetilde{C}_{2} z_{q_{m}} & \omega_{t} y_{1} & \ldots & \omega_{t} y_{t} & 0 & \ldots & 0
\end{array}\right|
\end{aligned}
$$

By combining (18), (19) with the relationship between $A_{1}$ and its determinant system, we give rise to the identity

$$
\begin{aligned}
\widetilde{D}_{m}^{n}\left(\begin{array}{cc}
\xi_{1}, \ldots, & \xi_{n} \\
z_{1}, \ldots, & z_{m}
\end{array}\right)= & k \sum_{p, q} \operatorname{sgn} p \operatorname{sgn} q D^{(2)_{m-m^{\prime}+t}^{n-n^{\prime}+t}}\left(\begin{array}{ccc}
\xi_{p_{1}} C_{1}, \ldots, & \xi_{p_{n-n^{\prime}}} C_{1}, \omega_{1}, \ldots, & \omega_{t} \\
z_{q_{1}}, & \ldots, & z_{q_{m-m^{\prime}+t}}
\end{array}\right) \times \\
& \times D^{(1)^{n^{\prime}}}\left(\begin{array}{ccc}
\xi_{p_{n-n^{\prime}+1}}, & \ldots, & \xi_{p_{n}} \\
\widetilde{C}_{2} z_{q_{m-m^{\prime}+t+1}}, \ldots, & \widetilde{C}_{2} z_{q_{m}}, y_{1}, \ldots, & y_{t}
\end{array}\right),
\end{aligned}
$$

where $k=1$ or $k=-1$. In view of (14), Lemma 3.3 implies that

$$
C_{2}=\widetilde{C}_{2}+\sum_{i=1}^{t} y_{i} \cdot \eta_{n^{\prime \prime}-t+i}^{\prime \prime}+\sum_{i=1}^{t} \sum_{j=1}^{m^{\prime \prime}}\left(\eta_{n^{\prime \prime}-t+i}^{\prime \prime} A_{2} u_{j}^{\prime \prime}\right) y_{i} \cdot \lambda_{j}^{\prime \prime},
$$

for some $\eta_{n^{\prime \prime}-t+i}^{\prime \prime} \in \mathbb{R}\left(B_{2}\right)(i=1, \ldots, t)$, is an arbitrary reflexive generalized inverse of $A_{2}$. Bearing in mind the bi-skew symmetry of $D_{m^{\prime}}^{(1)^{n^{\prime}}}$, we can substitute $C_{2}$ for $\widetilde{C}_{2}$ in (20). Since a determinant system for the fixed Fredholm operator is determined up to a constant (non-zero) factor, the sequence $\left(D_{m}^{n}\right)$ defined by (12) is a determinant system for $A_{2} A_{1}$. This completes the proof.

As a direct consequence of Theorem 3.4, we obtain the following result.

Corollary 3.5. Under the assumptions of Theorem 3.4, with $d^{\prime}=d^{\prime \prime}=0$, the formula (12) is of Cauchy-Binet type.

\section{Conclusions}

In the paper, products of Fredholm operators acting between arbitrary linear spaces were considered. By exploiting terms of determinant systems for operators 
$A$ and $B$, with $A B$ well-defined, we provided a direct construction of a determinant system for $A B$. The obtained result leads to a generalization of the Cauchy-Binet formula to Fredholm operators and yields an important tool for solutions of problems in various branches of applied science and engineering.

\section{References}

[1] Leżański T., The Fredholm theory of linear equations in Banach spaces, Stud. Math. 1953, 13, 244-276.

[2] Sikorski R., On Leżański's determinants of linear equations in Banach spaces, Stud. Math. 1953, 14, 24-48.

[3] Sikorski R., Determinant systems, Stud. Math. 1959, 18, 161-186.

[4] Sikorski R., The determinant theory in Banach spaces, Colloq. Math. 1961, 8, 141-198.

[5] Buraczewski A., The determinant theory of generalized Fredholm operators, Studia Math. 1963, 22, 265-307.

[6] Buraczewski A., Sikorski R., Analytic formulae for determinant systems in Banach spaces, Studia Math. 1980, 67, 85-101.

[7] Buraczewski A., Determinant system for composite of generalized Fredholm operators, Studia Math. 1970, 34, 197-207.

[8] Marcus M., Minc H., Introduction to Linear Algebra, Dover Publications, New York 1988.

[9] Lancaster P., Tismenetsky M., The Theory of Matrices. Computer Science and Applied Mathematics, Academic Press, New York 1985.

[10] Shafarevich I.R., Remizov A.O., Linear Algebra and Geometry, Springer-Verlag, Berlin 2012.

[11] Brualdi R.A., Schneider H., Determinantal identities: Gauss, Schur, Cauchy, Sylvester, Kronecker, Jacobi, Binet, Laplace, Muir, and Cayley, Linear Algebra Appl. 1983, 52/53, 769-791.

[12] Vishwanathan S.V.N., Smola A.J., Vidal R., Binet-Cauchy kernels on dynamical systems and its application to the analysis of dynamic scenes, Int. J. Comput. Vision 2007, 73(1), 95-119.

[13] Konstantopoulos T., A multilinear algebra proof for the Cauchy-Binet formula and a multilinear version of Parseval's identity, Linear Algebra Appl. 2013, 439 (9), 2651-2658.

[14] Karlin S., Rinott Y., A generalized Cauchy-Binet formula and applications to total positivity and majorization, J. Mult. Anal. 1988, 27, 284-299.

[15] Shevelev V., Combinatorial minors for matrix functions and their applications, Zeszyty Naukowe Politechniki Śląskiej 2014, seria: Matematyka Stosowana, 4, 5-16.

[16] Caracciolo S., Sokal A.D., Sportiello A., Noncommutative determinants, Cauchy-Binet formulae and Capelli-type identities. I. Generalizations of the Capelli and Turnbull identities, Electron. J. Comb. 2009, 16 (1), Research Paper R103, 1-43.

[17] Knill O., Cauchy-Binet for pseudo-determinants, Linear Algebra Appl. 2014, 459, 522-547.

[18] Ciecierska G., Determinant systems for nuclear perturbations of Fredholm operators in Frechet spaces, PanAmer. Math. J. 2014, 24(1), 1-20.

[19] Ciecierska G., Formulas of Fredholm type for Fredholm linear equations in Frechet spaces, Math. Aeterna 2015, 5(5), 945-960.

[20] Ciecierska G., Determinant systems method for computing reflexive generalized inverses of products of Fredholm operators, Math. Aeterna 2016, 6(6), 895-906.

[21] Ruston A.F., Fredholm theory in Banach spaces, Cambridge Tracts in Math. 86, Cambridge Univ. Press, Cambridge 1986.

[22] Ben-Israel A., Greville T.N.E., Generalized Inverses. Theory and Applications, Springer-Verlag, New York 2003. 